\title{
VIEWPOINT
}

\section{Radiation Therapy for Rectal Cancer: Toward an Evidence-based Approach Suitable to the Region}

\author{
JM Plummer ${ }^{1}$, S Ward-Chin ${ }^{2}$, PO Roberts ${ }^{1}$
}

\section{INTRODUCTION}

Colorectal cancer is the third most common cancer in both genders in the region with the rectum being the most common named site for cancer development. A significant number of these patients present with locally advanced disease (T3/4 or nodal involvement) (1). Compared with other parts of the colorectum, patients with cancer of the middle and lower rectum are at high risk of local and systemic failure and death even with skilled surgery. There is an important role for radiotherapy in improving outcome from this cancer and the Swedish Rectal Cancer Trial clearly established the benefits of neo-adjuvant short course radiotherapy (SCR). These are reduced local and systemic recurrence and improved survival (2). This approach has also been shown to be superior to postoperative radiotherapy (3). This regime of radiotherapy (SCR) is given in the form of 5 Gy for five consecutive days for a total of 25 Gy followed by immediate surgery the following week.

Short course radiotherapy is not universally practised and the majority of North American oncology centres will offer these same patients conventional chemoradiation (CCR) for a total of 50.4 Gy given as 1.8 Gy per day for 28 days followed by surgery four to six weeks later. During the period of the radiation, patients also receive radiosensitizing 5-flurouracil or capecitabine. Compared to SCR, this CCR will have more clinical and pathological complete response (4), and although there is a theoretical benefit of more sphincter sparing resections, it is yet to be proven by randomized trials (5).

Importantly, there is an increasing body of literature which shows that compared to CCR, SCR has similar long term local recurrence and survival $(4,6-7)$. This is the most important outcome to the majority of patients, hence quali-

Keywords: Caribbean, colorectal cancer, conventional chemoradiation, short course radiotherapy

From: ${ }^{1}$ Department of Surgery, Radiology, Anaesthesia and Intensive Care, and ${ }^{2}$ Department of Medicine, The University of the West Indies, Kingston 7, Jamaica.

Corespondence: Dr JM Plummer, Department of Surgery, Radiology, Anaesthesia and Intensive Care, The University of the West Indies, Kingston

7, Jamaica. E-mail: joseph_plummer@yahoo.com fied patients will do equally well with either approach from an oncology point of view. But the SCR also has the advantage of having greater compliance and less acute toxicity (4). Short course radiotherapy is also less expensive and it frees up the radio-oncology unit such that more patients can be accommodated for radiotherapy.

This is critical in Jamaica and other developing countries that lack enough radiotherapy machines or sufficient numbers of specialized doctors and other health professionals to adequately treat the majority of cancer victims who can benefit (8). The majority of our patients received radiotherapy through the cobalt Radiotherapy Units at the Kingston Public Hospital (KPH) and a smaller unit at the Cornwall Regional Hospital (CRH). These units provide the only affordable source of radiation as few can afford the linear accelerator at the privately run facility present on the island. In addition to rectal cancers, cancers of the head and neck, breast, lung, genitourinary (including cervix and prostate), skin, lymphomas and metastatic disease are routed through these units, which meet only $35-40 \%$ of the daily demands (9), with the result being unacceptable long waiting times, sometimes approaching a year (10) and less than the desirable oncologic outcome which is acknowledged by the patients and healthcare workers.

While private/public partnerships have been proposed (10), other creative means have to be found in an attempt to improve rectal cancer care. One method can be to develop our own protocol for rectal cancer patients as has been done in some centres with good effect (11). In India, for example, there has been the call for an abbreviated protocol which may be more economically suited for that country (12). Another approach must be a re-examination of the CCR protocol which is currently being employed at all our local radiotherapy units, with a view to offering SCR to some of our operable T3 rectal cancer patients. This will reduce the demands on these facilities, thus allowing the provisions of the necessary neo-adjuvant radiotherapy to more patients in a timely manner whilst not compromising the quality of care being delivered. It is hoped that once this change has been successfully implemented, a part of a long term soultion must also be evidence-based re-examination of all radiotherapy protocols for the other various cancers competing for this scarce resource in an attempt to improve patient outcome. 


\section{REFERENCES}

1. McFarlane ME, Rhoden A, Fletcher PR, Carpenter R. Cancer of the colon and rectum in a Jamaica population: diagnostic implications of the changing frequency and subsite distribution. West Indian Med J 2004; 53: 170-3.

2. Swedish Rectal Cancer Trial. Improved survival with preoperative radiotherapy in resectable rectal cancer. N Engl J Med 1997; 336: 980 7.

3. Sauer R, Becker H, Hohenberger W, Rodel C, Wittekind C, Fietkau R et al. Preoperative versus postoperative chemoradiotherapy for rectal cancer. N Engl J Med 2004; 351: 1731-40.

4. Bujko K, Nowacki MP, Nasierowska-Guttmejer A, Michalski W, Bebenek M, Kryj M. Long-term results of a randomized trial comparing preoperative short-course radiotherapy with conventionally fractionated chemoradiation for rectal cancer. B J Surgery 2006; 93: 1215-23.

5. Gerard JP, Rostom Y, Gal J, Benchimol D, Ortholan C, Aschele C et al Can we increase the chance of sphincter saving surgery in rectal cancer with neoadjuvant treatments: lessons from a systematic review of recent randomized trials. Crit Rev Oncol Haematol 2012; 81: 21-8.

6. Siegel R, Burock S, Wernecke K-D, Kretzschmar A, Dietel M, Loy V et al. Preoperative short-course radiotherapy versus combined radiochemotherapy in locally advanced rectal cancer: a multi-centre prospectively randomised study of the Berlin Cancer Society. BMC Cancer 2009; 9: 50.

7. Ngan SY, Burmeister B, Fisher RJ, Solomon M, Goldstein D, Joseph D et al. Randomized trial of short-course radiotherapy versus long-course chemoradiation comparing rates of local recurrence in patients with T3 rectal cancer: Trans-Tasman Radiation Oncology Croup trial 01.04. J Clin Oncol 2012; 30: 3827-33. Epub 2012 Sept 24.

8. Cancer treatment in developing countries [monograph on the internet]. International Atomic Energy Agency; 2009 [cited 2012 Oct 31]. Available from: http://www.iaea.org

9. Hines H. Cancer cases increasing at Cornwall Regional HospitalFerguson. Jamaica Observer. 2012 March 29: News. Available from: http://www.jamaicaobserver.com/westernnews/Cancer-casesincreasing-at-Cornwall-Regional-Hospital_Fegurson_11153944

10. Clarke F. Best alternative for cancer patients. Jamaica Gleaner. 2012 Jan 22: News. Available from: http://jamaica-gleaner.com/gleaner/ 20120122/lead/lead41.html

11. Kube R, Ptok H, Jacob D, Fahlke J, Mroczkowski P, Lippert H et al. Modified neoadjuvant short-course radiation in uT3 rectal carcinoma: low local recurrence rate with unchanged overall survival and frequent morbidity. Int J Colorectal Dis 2010 Jan; 25: 109-17.

12. Bansal V, Bhutani R, Doval D, Kumar K, Pande P, Kumar G. Neo adjuvant chemo-radiotherapy and rectal cancer: Can India follow the West? J Can Res Ther 2012; 8: 209-14. 